\title{
Nanopores formed by DNA origami: a review
}

\author{
Nicholas A. W. Bell and Ulrich F. Keyser ${ }^{\mathrm{a}}$ \\ ${ }^{a}$ Cavendish Laboratory, Cambridge University, J J Thomson Avenue, Cambridge, CB3 OHE

\section{$\underline{\text { Abstract }}$}

Nanopores have emerged over the past two decades to become an important technique in single molecule experimental physics and biomolecule sensing. Recently DNA nanotechnology, in particular DNA origami, has been used for the formation of nanopores in insulating materials. DNA origami is a very attractive technique for the formation of nanopores since it enables the construction of 3D shapes with precise control over geometry and surface functionality. DNA origami has been applied to nanopore research by forming hybrid architectures with solid state nanopores and by direct insertion into lipid bilayers. This review discusses recent experimental work in this area and provides an outlook for future avenues and challenges.

\section{Keywords}

Nanopores

DNA origami

Self-assembly

Single molecule

Nanotechnology 


\section{Introduction}

Nanopores are a relatively new technique for detecting single molecules in solution and are garnering great interest due to their applications in areas such as DNA sequencing [1,2]. The basic idea for single molecule sensing is to apply a voltage across a single nanoscale pore separating two electrolyte solutions and measure the resulting ionic current. Individual molecules are detected as they translocate the pore since they reduce the flow of ions and therefore the measured current. Seminal research in the 1990s first showed that single molecules such as nucleic acids could be detected in this way [3-5]. In the past two decades nanopores have been applied to the detection of a wide range of biomolecules such as DNA, RNA and proteins - see M. Wanunu [6] for a recent review of biomolecule detection with nanopores. Nanopores have also uncovered a wealth of interesting phenomena covering diverse research topics such as nanoscale electrokinetics [7], polymer physics [8] and surface chemistry [9].

Nanopore research has traditionally been split into two disciplines: solid state nanopores and biological nanopores. Solid state nanopores are entirely synthetic and can be fabricated by techniques such as ion beam drilling of thin insulating membranes [10] or laser assisted pulling of glass capillaries [11-14]. Biological nanopores are made by reconstitution of a membrane spanning protein into a lipid bilayer $[4,15]$. A crucial aspect of solid state and biological nanopore sensing is the ability to control both the geometry and surface functionality of the nanopore. Nanopore geometry determines the signal of measurements and enables selectivity based on physical size. Surface functionality allows for chemical specificity in nanopore experiments.

First we consider aspects of nanopore geometry. Advances in fabrication techniques have recently enabled the production of solid state nanopores with similar dimensions to biological nanopores such as alpha-hemolysin [16] and atomically thin nanopores can be made in graphene [17]. However atomic reproducibility of solid state nanopore 3D profiles is still not achievable and small (below $10 \mathrm{~nm}$ ) diameters also require time consuming transmission electron microscopy (TEM) techniques. Biological nanopores are atomically reproducible, however they are mostly limited to studying single stranded DNA or smaller biomolecules there being only a few examples of engineered non-gating pores with diameters wider than $2 \mathrm{~nm}$ that show DNA translocation $[18,19]$. The most widely used biological nanopore, alpha-hemolysin, is $1.4 \mathrm{~nm}$ at its narrowest constriction [20]. In contrast, solid state nanopores can be tuned to arbitrarily large diameters with nanometre accuracy and therefore are useful for testing larger biomolecules such as double stranded DNA and proteins [21].

The second aspect of control for nanopore sensing is surface functionality. Biological nanopores significantly outperform their solid state counterparts when it comes to the ability to engineer surface functionality. Genetic engineering of biological nanopores allows for the precise positioning of chemical groups which bind to analytes. In the first protein nanopore experiments of the $1990 \mathrm{~s}$ molecules simply passed through the nanopore driven under an electric field [3]. In later experiments, the introduction of engineered binding sites in a protein nanopore was used to create stochastic blockades as individual molecules bind on and off thereby modulating the ionic current [9]. This has enabled the specific detection of analytes such as ions [22], small organic molecules [23] and DNA bases [24]. Solid state nanopores can be chemically modified with monolayers $[25,26]$ however the precise positioning of single binding sites remains extremely challenging.

Having considered these aspects of nanopore geometry and surface functionality it is apt to ask what other nano-engineering techniques are available for fabricating isolated three-dimensional structures at the scale of a few to tens of nanometres? A method that has recently gathered 
significant momentum has been the design of nanoscale objects self-assembled from DNA. DNA follows simple rules of base pairing and synthetic DNA strands can be designed to form nanoscale structures. This field of DNA nanotechnology has been pioneered by Ned Seeman who first realised its potential in the 1980s [27]. A significant leap forward in the sophistication of designed DNA nanostructures was so-called "DNA origami" first described in 2006 by Paul Rothemund [28]. The DNA origami method is to take a long single "scaffold" strand and fold it into a desired shape using hundreds of short "staple" strands. The scaffold strand is often the genome of the m13mp18 virus and the staple strands are made by chemical synthesis. The base sequences of the synthetic strand hybridise at specific positions on the scaffold strand and thereby fold it into the designed nanostructure with a typical molecular weight of $\sim 5 \mathrm{MDa}$. DNA origami immediately opened up new avenues for DNA nanotechnology due to its simplicity and high yield. Previous work on DNA nanostructures had nearly always used short synthetic oligonucleotides as the only building block and very tight controls of stoichiometry and purification were needed to get reasonable yields. DNA origami overcomes issues of matched stoichiometry since the staple strands can be used in large excess to the scaffold. This also allows the full length staples to preferentially bind at their designed positions and remove potential secondary structure [28]. Modifications on the synthetic single strands can be used for site specific attachments on DNA origami for instance with gold nanoparticles [29,30], proteins [31] and fluorophores [32]. Readers are referred to several reviews for further information on the DNA origami method [33-36].

DNA origami represents an attractive method for forming nanopores since it can be used to fabricate 3D shapes with nanoscale level accuracy in geometry and surface properties. DNA origami can be used to form structures such as plates [28], cubes [37], curved beams [38] and shapes with complex curvatures [39]. Functional groups can be reliably positioned in a design with an accuracy of a few nanometres [40]. Recently a molecular model of a DNA origami design was made by cryo-EM imaging and this shows that the field can progress towards even greater positional accuracy [41]. Billions of nearly identical copies are created in parallel since the method relies on self-assembly. Finally we also add that the design of DNA origami structures is simple using freely available software such as caDNAno [42] and annealing protocols for synthesising DNA origami are typically less than a day.

DNA origami has been applied to nanopore research in two distinct ways -1 ) by trapping a DNA origami structure at the mouth of a solid state nanopore and 2) by insertion of a DNA origami structure, coated with hydrophobic moieties, into a lipid bilayer. Recent work in these two branches is now discussed.

\section{Hybrid nanopores formed by trapping DNA origami onto a solid state nanopore}

The combination of DNA origami and solid state nanopores was first described in 2012 [43,44]. The method was to make a hybrid nanopore by inserting a single engineered DNA origami into a solid state nanopore using the electrophoretic force. This idea is similar to that used by Hall et al [45] in 2010 for guiding a protein nanopore, alpha-hemolysin, into a solid state nanopore and thereby forming a hybrid architecture. This system combines the advantages of atomically engineered nanoconstructs and a substrate that is significantly more robust than a lipid bilayer.

Our work [43] used a funnel shaped DNA origami designed with a narrowest constriction of $3 \times 3$ double helix widths $(7.5 \mathrm{~nm} \times 7.5 \mathrm{~nm})$ and a double stranded DNA tail 2.3 kilobasepair (kbp) long to aid trapping in the correct orientation (Fig. 1a,b). These DNA origami structures were added in solution and a voltage applied with a polarity to attract the negatively charged DNA origami towards the solid state nanopore. A characteristic decrease in ionic current was observed after a few 
seconds of applying the voltage (Fig. 1C). This corresponds to a single DNA origami being trapped into the solid state nanopore and thereby reducing the flow of ions. The trapping process was found to be highly controllable and reversible. The DNA origami could be simply expelled from the solid state nanopore by switching the polarity of the potential and therefore the direction of the electrophoretic force (Fig. 1d). Another DNA origami could then be assembled by again reversing the potential. This process could be performed many times without significant clogging of the bare solid state nanopore. The waiting time between trapping events can be easily tuned by adjusting the applied voltage or the DNA origami concentration. This means there are several parameters that can be adjusted in order to quickly obtain a single insertion.

(a)

(b)
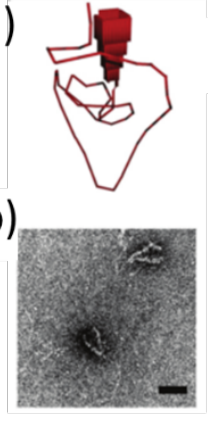

(e)

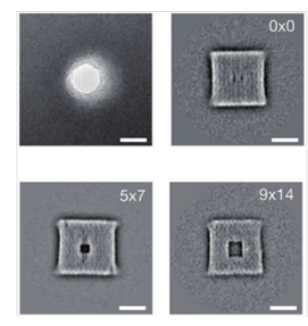

(c)

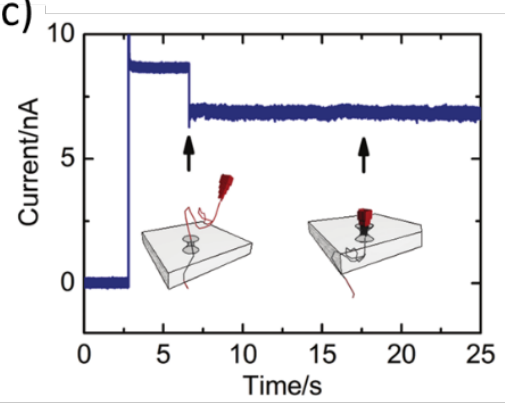

(d)

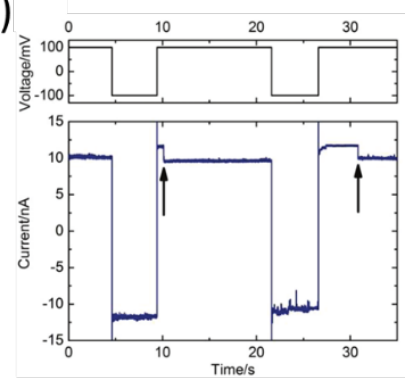

(f)

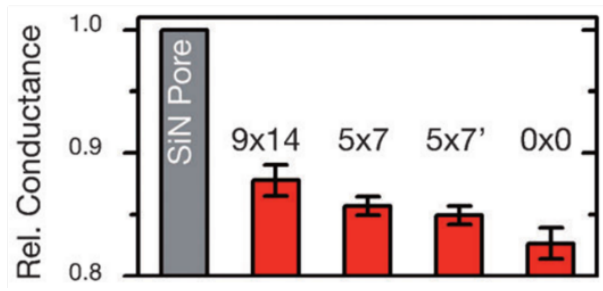

Figure 1 - (a) Rendered design of a funnel shaped DNA origami designed to be trapped in a solid state nanopore. The funnel is approximately $51 \mathrm{~nm}$ long, $22.5 \mathrm{~nm}$ wide at the base and $7.5 \mathrm{~nm}$ wide at the tip with a $2.3 \mathrm{kbp}(\approx 800 \mathrm{~nm})$ long tail. (b) Transmission electron microscopy images of assembled DNA origami structures. Scale bar $=40 \mathrm{~nm}$. (c) Formation of a hybrid nanopore. $A$ voltage of $+100 \mathrm{mV}$ is applied at $t=2.8 \mathrm{~s}$ and at $t=6.2 \mathrm{~s}$ a sudden drop indicates trapping of the DNA origami. In this case the diameter of the solid state nanopore was $13 \mathrm{~nm}$. (c) The trapping is reversible - by switching to a negative potential for a short time the DNA origami is ejected and after returning to a positive potential a DNA origami is quickly trapped again. (e) TEM images (class averages) showing designs of DNA origami nanoplates with different aperture sizes. Each nanoplate design has a thickness of $6 \mathrm{~nm}$. Scale bars $=20 \mathrm{~nm}$. (f) Relative conductance (defined as the conductance of the hybrid nanopore divided by the conductance of the bare solid state nanopore) for the designs shown in Figure (e). Figures 1(a)-(d) reprinted with permission from [43], copyright (2012) American Chemical Society. Figures 1(e)-(f) reprinted with permission from [44], copyright (2012) John Wiley and Sons.

Wei et al [44] reported experiments on DNA origami nanoplates with a thickness of $6 \mathrm{~nm}$ and varying pore diameters (Fig. 1e). A hybrid architecture was also formed by electrophoretic guiding of the DNA origami into a single solid state nanopore. They found that the relative conductance of the nanoplate decreased as the pore size was reduced giving evidence that the nanoplate was trapped in the correct orientation (Fig. 1f). They also showed ionic current traces that suggest that the DNA origami could be used as a size selective filter since proteins larger than the aperture of the nanoplate did not translocate through. 
Several challenges are immediately apparent from the first two papers of Bell et al and Wei et al. Simple geometrical models of the systems showed that the ionic flow was substantially higher than what would be expected if the DNA origami was completely insulating. This is somewhat surprising given the dense packing of helices suggested by TEM images (Fig. 1b, 1e) but shows that there is substantial leakage current through the structure and its seal. Therefore the signals for translocating molecules through the hybrid nanopore are not as high as might be hoped. Also there can be a significant increase in ionic current noise when the hybrid nanopore is formed compared to the bare solid state nanopore. This fluctuating current is also highly variable for different DNA origami trappings (on the same solid state nanopore). This is clearly undesirable for single molecule sensing however it can be ameliorated to some degree by exchanging DNA origami structures until a low noise hybrid nanopore is achieved. The increase in current noise upon hybrid nanopore formation may be explained by the fluctuations of the DNA origami. The source of the wide variability in noise levels is still uncertain but one can speculate that this is due to different conformations in which the DNA origami is trapped or small differences between individual DNA origami structures such as missing staple strands or slightly misfolded structures. Some possible solutions to these challenges are discussed in the outlook at the end of this review.

Subsequent to these first two papers, our group showed that DNA origami nanoplates could also be combined with nanocapillaries with final diameters of a few tens of nanometres [46]. Nanocapillaries are a simple method of solid state nanopore fabrication made by laser assisted capillary pulling which can be easily multiplexed into arrays [11]. The advantage of this system is the quick and cheap fabrication of the nanocapillaries and also the geometry of the nanocapillary allows for simultaneous optical imaging alongside ionic current measurements. This was exploited by labelling the DNA origami with fluorophores so that the trapping process could be imaged at the same time as ionic current measurements (Fig. 2a). It was also found with this system that a smaller pore size in the DNA origami influences the translocation of DNA. A pore size of $5 \mathrm{~nm} \times 7 \mathrm{~nm}$ reduces the number of DNA strands that can translocate with hairpins compared to a DNA origami with a pore size of $14 \mathrm{~nm} \times 15 \mathrm{~nm}$ (Fig. 2b). 
(a)

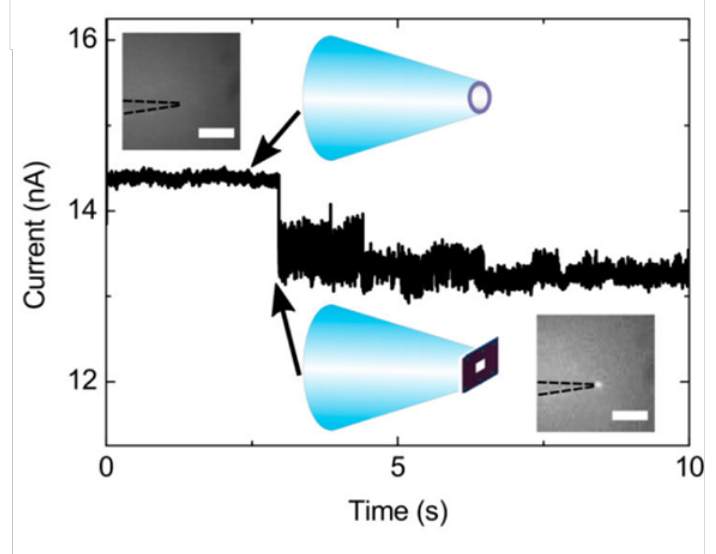

(b)
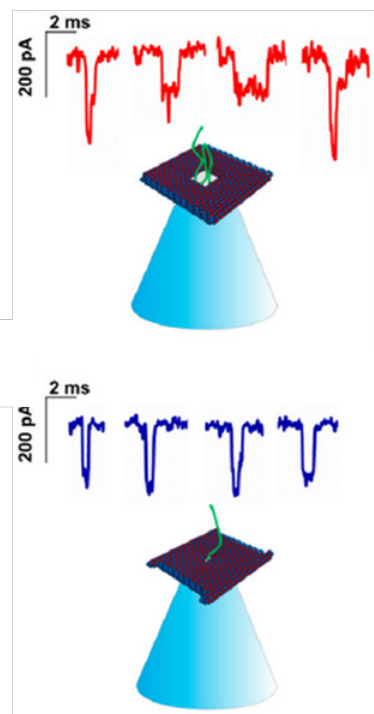

(c)

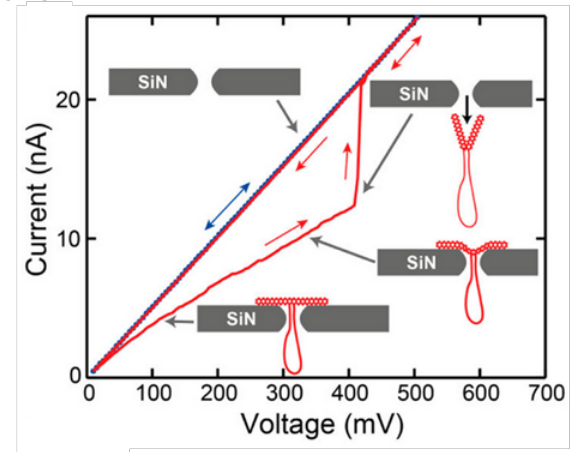

(d)

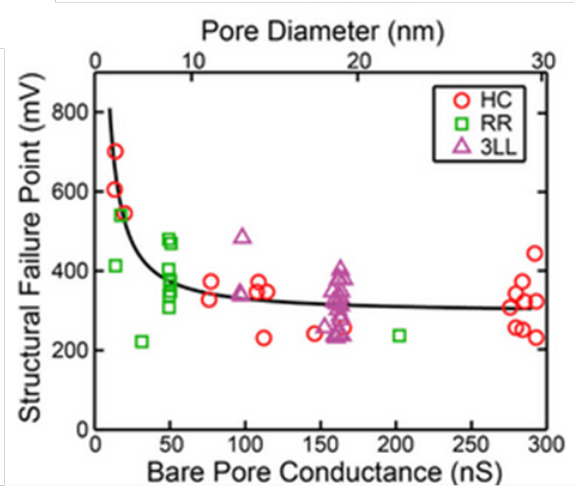

Figure 2 - (a) Trapping of a DNA origami nanoplate onto a nanocapillary showing the characteristic ionic current drop. This particular design was tagged with six Cy3 fluorophores. This enabled simultaneous optical imaging of the trapping as shown by the inset images. Scale bars $=5 \mu \mathrm{m}$. (b) DNA translocation through the hybrid nanopores showed that the size of the aperture influences the DNA folding state as it passes through. (c) Voltage ramping can be used to probe the structural rigidity of different origami designs by measuring the voltage at which the DNA origami is sucked through the pore so that the current returns to the bare solid state nanopore value. The red line shows a typical IV curve after trapping a DNA origami and the return to the bare pore current at a certain threshold voltage. (d) Voltage of structural failure for a three layer honeycomb lattice design (red circles), a single layer DNA origami sheet (green squares) and a three layer square lattice design (purple triangles). Figures 2(a)-(b) reprinted with permission from [46], copyright (2013) American Chemical Society. Figures 2(c)-(d) reprinted with permission from [47], copyright (2014) American Chemical Society.

Two recent papers have further characterised the ionic currents observed after trapping different DNA origami structures and the behaviour at different voltages. Plesa et al [47] investigated the leakage currents through DNA origami structures with varying numbers of layers and without any aperture. A honeycomb lattice was found to have lower leakage current compared to square lattices with 1-3 helices. Surprisingly it was found that the relative conductance was slightly lower for a single layer square lattice layout compared to 2 and 3 layers. This was attributed to different arrangements of staple strands within the different structures but could also point to varying 
mechanical deformations of the DNA origami when forming a hybrid nanopore. This paper also investigated the effect of ramping the voltage after hybrid formation. A non-linear current-voltage curve was observed and attributed to mechanical buckling of the DNA origami as the voltage is increased (Fig. 2c). Increasing the voltage eventually causes the structure to collapse and translocate through the solid state nanopore (Fig. 2d).

Hernández-Ainsa et al [48] analysed the current reduction when a DNA origami nanoplate, with a $450 \mathrm{~nm}$ long double stranded DNA tail, was trapped onto a nanocapillary. Histograms of the current drop showed two distinct peaks whereas without the double stranded DNA tail only one peak was

seen. The number of DNA origami structures trapped in the two states was influenced by the trapping voltage with an increasing proportion in the lower (larger ionic current decrease) state at higher voltages. This two state behaviour was assigned to a deformation of the DNA origami above a certain voltage.

\section{DNA origami nanopores inserted into lipid bilayers}

The second distinct way in which DNA nanostructures have been applied to nanopore sensing is by insertion of a structure into a lipid bilayer. This mimics the behaviour of membrane proteins which insert into cell membranes for controlling the transport of molecules and ions. The charged phosphate backbone of DNA is hydrophilic and so the DNA nanostructure must be decorated with hydrophobic chemical groups to overcome the large free energy penalty of forming a hole in the lipid bilayer.

Langecker et al [49] attached 26 cholesterol tagged oligonucleotides to a stem and barrel DNA origami structure which formed a pore $2 \mathrm{~nm}$ wide and $42 \mathrm{~nm}$ in length (Fig. 3a). Transmission electron microscopy showed that these structures inserted into lipid vesicles with the desired orientation (Fig. 3b). Single channel electrophysiology experiments suggest the formation of channels with unitary conductance of $\mathrm{G}=0.87 \mathrm{nS}$ in the presence of $1 \mathrm{M} \mathrm{KCl}, 10 \mathrm{mM}$ Tris $(\mathrm{pH} 8)$, $2 \mathrm{mM} \mathrm{MgCl}_{2}$ (Fig. 3c). It should be noted that an advantage of DNA origami inserted into lipid bilayers is that a negative voltage can also be applied whereas in the hybrid solid state nanopore configuration this will cause the DNA origami to escape the solid state nanopore. The pores by Langecker et al showed variable noise similar to what has been observed with solid state hybrids. Several sub-conductance states were observed in some cases and the frequency of these subconductance states could be increased by modifying the pore so that a single staple strand extended into the pore interior. Langecker et al also gave evidence of translocations of single stranded DNA hairpins and G-quadruplexes as shown by blockades of ionic current (Fig. 3d). 
(a)

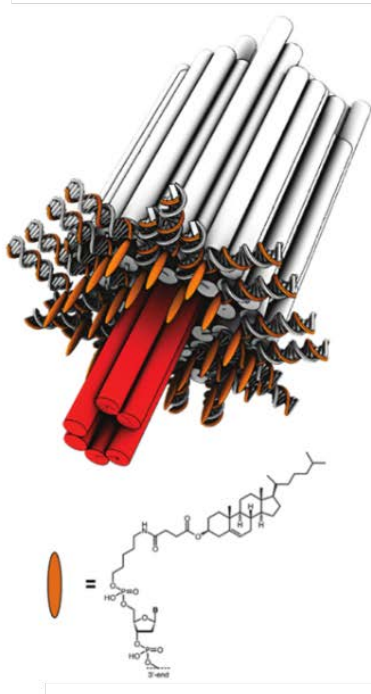

(c)

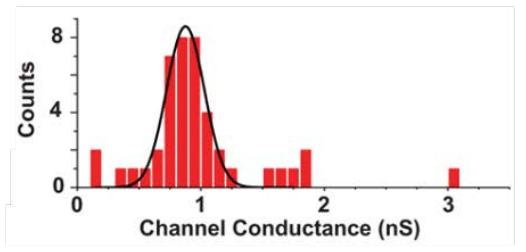

(b)

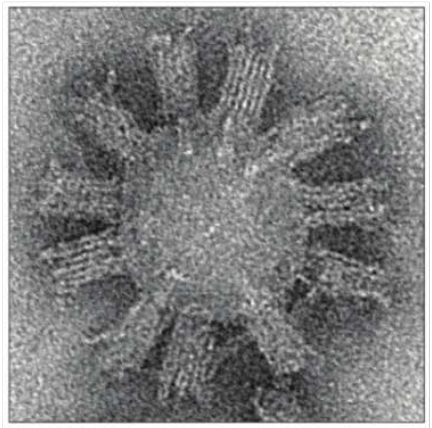

25nm

(d)

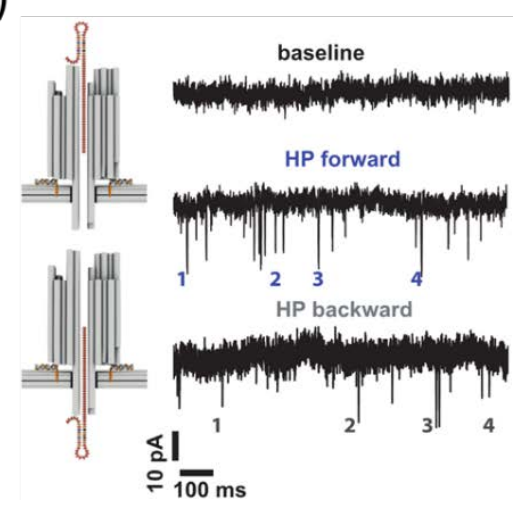

Figure 3 - Design and functionality of a synthetic lipid membrane channel made from DNA. (a) DNA origami design featuring a barrel and stem structure with 26 cholesterol modified oligonucleotides (represented in orange) surrounding the stem. (b) TEM image showing the assembly of the designed structures in lipid vesicles with the desired orientation. (c) Histogram of single channel conductance values from ionic current measurements in a solution of $1 \mathrm{M} \mathrm{KCl}, 2 \mathrm{mM} \mathrm{MgCl}_{2}$. (d) lonic current traces showing baseline current and translocation of DNA hairpins through the channel. Figures reprinted with permission from [49], copyright (2012) American Association for the Advancement of Science.

Burns et al [50] created a DNA based nanopore with the same $2 \mathrm{~nm}$ width but a shorter length of 15 $\mathrm{nm}$. This small nanopore did not use the DNA origami technique of folding a long scaffold but rather was made by mixing 14 synthetic nucleotides designed to form a hollow DNA tube. Ethyl modified phosphorothioate groups were substituted in place of phosphate groups at 72 selected positions in order to form a $2.2 \mathrm{~nm}$ long ring of hydrophobic motifs (Fig. 4a). The correct geometrical assembly was evidenced by atomic force microscopy (Fig. 4b) and dynamic light scattering. Ionic current measurements of these DNA nanopores in suspended lipid bilayers found a conductance peak at approximately $0.4 \mathrm{nS}$ in $1 \mathrm{M} \mathrm{KCl}, 50 \mathrm{mM}$ Tris (pH 5). In a subsequent paper Burns et al [51] showed that a similar sized DNA nanopore could also be inserted into a lipid bilayer using just two porphyrin tags instead of the ethyl-phosphorothioate modifications (Fig. 4d). A channel conductance of the same order of magnitude was reported (Fig. 4e). However, both these papers did not report any translocation of polymers through pores of similar diameter to the initial Langecker $e t$ al design. It remains to be determined if the much larger DNA origami structure of Langecker et al gives a higher stability to the DNA channel that allows for single stranded DNA translocation. Structural integrity is clearly important for these channels since it can be expected that the pore size of $2 \mathrm{~nm}$ is close to a 
limit where DNA translocation will not be possible given the strong negative charge of the pore ( $\mathrm{pK}_{\mathrm{a}}$ $\sim 2$ for DNA phosphate groups) and a Debye length of $0.3 \mathrm{~nm}$ in $1 \mathrm{M} \mathrm{KCl}$.

(a)

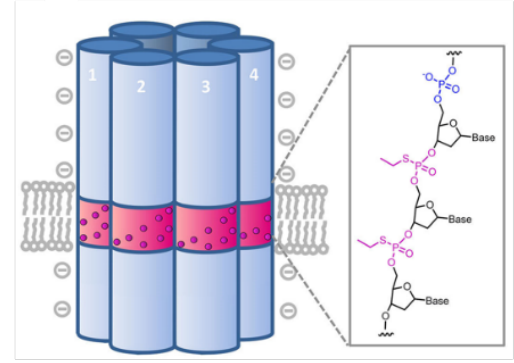

(d)

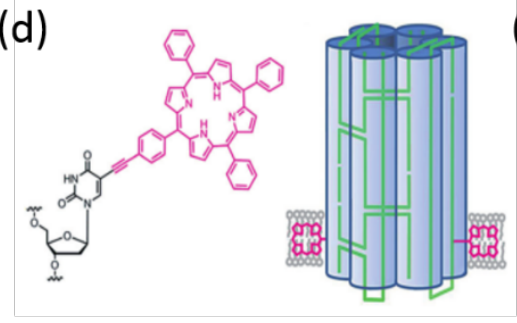

(b)

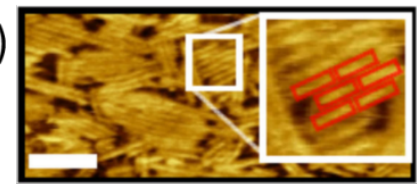

(c)

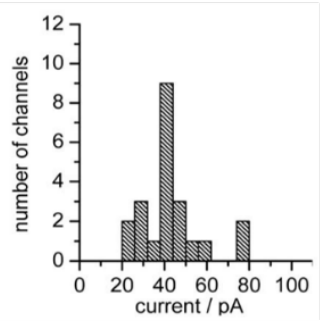

(e)

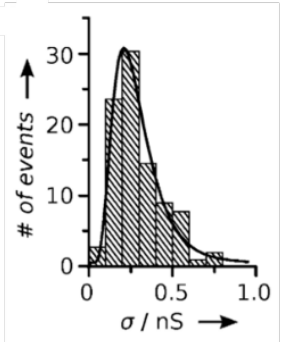

Figure 4 - (a) A DNA nanopore designed with ethyl-phosphorothioate modifications to provide hydrophobicity for insertion into a lipid bilayer. (b) Atomic force microscopy of DNA nanopores as designed in (a). Base stacking causes aggregates to be observed. Scale bar $=100 \mathrm{~nm}$. (c) Histogram of current levels observed at $+100 \mathrm{mV}$ in $1 \mathrm{M} \mathrm{KCl}, 50 \mathrm{mM}$ Tris $(\mathrm{pH} 5)$. The modal value is therefore at approximately $0.4 \mathrm{nS}$. (d) A similar sized structure to (a) but using just two porphyrin based tags for insertion rather than the 72 ethyl-phosphorothioate tags used in (a). (e) Histogram of conductance values for design (d) in $1 \mathrm{M} \mathrm{KCl}, 50 \mathrm{mM}$ Tris ( $\mathrm{pH}$ 8). Figures $4(\mathrm{a})$-(c) reprinted with permission from [50], copyright (2013) American Chemical Society. Figures 4(d)-(e) reprinted with permission from [51], copyright (2013) John Wiley and Sons.

\section{Outlook and challenges}

DNA nanotechnology and the origami method has created a significant leap forward in our ability to design complex nanometre scale objects by self-assembly. This technique is well suited for use in nanopore sensing where control of surface chemistry and geometry on the scale of single molecules is needed. The papers described in this review show the first exciting steps towards the goal of improving nanopore sensing using DNA nanostructures which can be adapted to different analytes.

The versatility of DNA based biochemistry means that we can envisage many potential avenues for future research. The detection of DNA translocation has already been reported. An extension of this would be to attach protein binding motifs, such as aptamers or antibody fragments, to DNA origami designs. This could be used as a basis for the stochastic sensing of specific proteins which has already been demonstrated with biological nanopores [52] but is difficult to achieve with solid state nanopores since without a binding site the translocation time is fast compared with the measurement bandwidth $[53,54]$. Another interesting possibility is to attach gold nanoparticles to create plasmonic nanopores where an enhanced fluorescence or Raman signal could be acquired for translocating molecules at the same time as ionic current [30]. Here, DNA origami self-assembly 
simplifies the alignment process of the plasmonic structure with respect to the nanopore and also would offer the possibility of simple exchange of DNA origami structures if one fails to work. This could be an advantage over modern nanofabrication techniques that recently demonstrated the alignment of gold nanostructures with nanopores [55].

DNA origami that insert into lipid bilayers might also be used for making adaptable nanopore sensors with diameters greater than typical biological nanopores such as alpha-hemolysin. The lipid bilayer inserting DNA nanopores shown in Figures 3 and 4 all have a pore formed by a hexagonal arrangement of DNA helices with a diameter of approximately $2 \mathrm{~nm}$. One could envisage making larger diameter DNA nanopores so that double stranded DNA and folded proteins could be translocated. The challenge for creating such a wide diameter nanopore (and also for increasing the low insertion efficiency of lipid bilayer spanning DNA nanopores already reported) will be to find chemical methods of making sufficiently hydrophobic patches on the DNA nanopore in order to overcome the energy penalty of pore formation in a lipid bilayer.

A key challenge for improving sensing with DNA nanopores is to reduce the leakage currents and thereby increase signals for translocating molecules. This is particularly true for the solid state nanopore hybrids where high leakage currents have been observed. Recent developments in DNA origami design with close packed hexagonal lattices may offer a reduction in current leakage [56]. Also DNA groove binders or high concentrations of multivalent ions could help to reduce the free space between helices and thereby leakage. Studies with varying the concentration of monovalent ions have already been performed by Plesa et al [47] but only showed a slight decrease in relative conductance with increasing $\mathrm{KCl}$ concentration. It will also be interesting to measure, in future experiments, whether the leakage current for DNA nanopores shows cation selectivity for small pores or structures without pores. We expect this to occur due to the negative charge of the phosphate groups of the DNA backbone.

Another challenge that should not be overlooked is the degree of possible mechanical deformation of the DNA origami structure. The electric field strength is on the order of $10^{7} \mathrm{~V} / \mathrm{m}$ in typical nanopore measurements which likely creates some distortion of the highly charged DNA origami structure and means that AFM and TEM images are not necessarily indicative of the shape of the DNA origami when it is trapped at the nanopore. Further modelling is needed to predict how this electric field affects the shape of the DNA origami. This could help to explain the large amplitude noise fluctuations observed for DNA origami nanopores. Experiments combining optical tweezers [57] and nanopores could also help in this regard by attaching a DNA origami structure to a colloid and measuring the forces on it as it is moved to the entrance of a solid state nanopore.

The molecular basis for insertion of DNA nanopores into lipid bilayers is another area that requires more understanding as a basis for future development. Presumably the DNA nanopores are initially anchored onto the lipid bilayer by one or a few of their hydrophobic moieties. Fluorescence imaging and electron microscopy clearly shows that DNA nanostructures will efficiently anchor to the surfaces of lipid vesicles with just a few cholesterol or porphyrin modified oligonucleotides $[51,58]$. The formation of a pore then likely has a high activation barrier since it involves significant rearrangement of the lipid bilayer to allow the other hydrophobic moieties of the DNA nanopore to anchor in and simultanesously form a pore. Indeed Langecker et al [49] reported that voltage pulses were needed to insert their DNA nanopores and all the reported papers have low pore insertion efficiencies judging by the concentration of DNA nanopores needed to form a single bilayer spanning pore. It is likely then that many DNA nanopores become attached to the bilayer before one forms a pore. Also the arrangement of lipids in the vicinity of the DNA nanopore is not known and therefore it is not clear whether these DNA nanopores in fact span the bilayer or rather stabilise a toroidal lipid 
pore [59]. Langecker et al [49] showed transmission electron microscope images which suggest their pores likely span the lipid bilayer but these images were made with a different lipid to that used for electrical measurements which could influence the position of the pore in the bilayer. Molecular dynamics simulations may help to answer some of these open questions by revealing the possible structural arrangements of lipid around these different DNA nanopores. Also combined fluorescence and electrical measurements may help to reveal the mechanisms and kinetics of anchorage into the lipid membrane and pore formation [60].

In addition to nanopore sensing we anticipate that DNA nanostructures could be used to mimic the behaviour of transmembrane proteins such as gating ion channels and active transporters. Walkers based on DNA origami [61] and DNA origami that responds to environmental cues [62,63] have already been reported in the literature and these ideas could be adapted to a nanopore design. An interesting possibility is also to combine DNA origami nanopores with synthetic tissues [64] to form sophisticated ion based circuits. Together, these intriguing ideas show that the field has great potential for future work.

\section{Acknowledgements}

The authors thank Sandip Ghosal, Catalin Chimerel and Vivek Thacker for critical reading of the manuscript. NAWB acknowledges funding from the EPSRC NanoDTC program and an EPSRC doctoral prize award, UFK acknowledges funding from an ERC starting grant.

1 Branton D, Deamer DW, Marziali A, Bayley H, Benner SA, Butler T, Di Ventra M, Garaj S, Hibbs A, Huang X, Jovanovich SB, Krstic PS, Lindsay S, Ling XS, Mastrangelo CH, Meller A, Oliver JS, Pershin Y V, Ramsey JM, Riehn R, Soni G V, Tabard-Cossa V, Wanunu M, Wiggin M \& Schloss JA (2008) The potential and challenges of nanopore sequencing. Nat. Biotechnol. 26, 1146-1153.

2 Pennisi E (2012) Search for Pore-fection. Science 336, 536-537.

3 Kasianowicz JJ, Brandin E, Branton D \& Deamer DW (1996) Characterization of individual polynucleotide molecules using a membrane channel. Proc. Natl. Acad. Sci. U. S. A. 93, 1377013773.

4 Bayley H \& Martin C (2000) Resistive-Pulse Sensing-From Microbes to Molecules. Chem. Rev. 100, 2575-2594.

5 Deamer DW \& Branton D (2002) Characterization of Nucleic Acids by Nanopore Analysis. Acc. Chem. Res. 35, 817-825.

6 Wanunu M (2012) Nanopores: A journey towards DNA sequencing. Phys. Life Rev. 9, 125-58.

7 Keyser UF, van Dorp S \& Lemay SG (2010) Tether forces in DNA electrophoresis. Chem. Soc. Rev. 39, 939-47.

8 Muthukumar M (2009) Polymer Translocation CRC Press.

9 Howorka S \& Siwy Z (2009) Nanopore analytics: sensing of single molecules. Chem. Soc. Rev. 38, 2360-2384.

10 Li J, Stein D, McMullan C, Branton D, Aziz MJ \& Golovchenko JA (2001) lon-beam sculpting at nanometre length scales. Nature 412, 166-169. 
11 Bell NAW, Thacker V V, Hernández-Ainsa S, Fuentes-Perez ME, Moreno-Herrero F, Liedl T \& Keyser UF (2013) Multiplexed ionic current sensing with glass nanopores. Lab Chip 13, 185962.

12 Piper JD, Li C, Lo C-J, Berry R, Korchev Y, Ying L \& Klenerman D (2008) Characterization and Application of Controllable Local Chemical Changes Produced by Reagent Delivery from a Nanopipet. J. Am. Chem. Soc. 130, 10386-10393.

13 Steinbock LJ, Otto O, Chimerel C, Gornall J \& Keyser UF (2010) Detecting DNA folding with nanocapillaries. Nano Lett. 10, 2493-7.

14 Gong X, Patil A V, Ivanov AP, Kong Q, Gibb T, Dogan F, deMello AJ \& Edel JB (2014) Label-free inflow detection of single DNA molecules using glass nanopipettes. Anal. Chem. 86, 835-41.

15 Bezrukov S (2000) Ion channels as molecular Coulter counters to probe metabolite transport. J. Membr. Biol. 174, 1-13.

16 Venta K, Shemer G, Puster M, Rodríguez-Manzo JA, Balan A, Rosenstein JK, Shepard K \& Drndić M (2013) Differentiation of short, single-stranded DNA homopolymers in solid-state nanopores. ACS Nano 7, 4629-36.

17 Garaj S, Hubbard W, Reina A, Kong J, Branton D \& Golovchenko JA (2010) Graphene as a subnanometre trans-electrode membrane. Nature 467, 190-3.

18 Wendell D, Jing P, Geng J, Subramaniam V, Lee TJ, Montemagno C \& Guo P (2009) Translocation of double-stranded DNA through membrane-adapted phi29 motor protein nanopores. Nat. Nanotechnol. 4, 765-72.

19 Franceschini L, Soskine M, Biesemans A \& Maglia G (2013) A nanopore machine promotes the vectorial transport of DNA across membranes. Nat. Commun. 4, 2415.

20 Song L, Hobaugh MR, Shustak C, Cheley S, Bayley H \& Gouaux JE (1996) Structure of staphylococcal $\alpha$-hemolysin, a heptameric transmembrane pore. Science 274, 1859-1866.

21 Dekker C (2007) Solid-state nanopores. Nat. Nanotechnol. 2, 209-215.

22 Braha O, Walker B, Cheley S, Kasianowicz JJ, Song L, Gouaux JE \& Bayley H (1997) Designed protein pores as components for biosensors. Chem. Biol. 4, 497-505.

23 Guan X, Gu L-Q, Cheley S, Braha O \& Bayley H (2005) Stochastic sensing of TNT with a genetically engineered pore. Chembiochem 6, 1875-1881.

24 Clarke J, Wu H-C, Jayasinghe L, Patel A, Reid S \& Bayley H (2009) Continuous base identification for single-molecule nanopore DNA sequencing. Nat. Nanotechnol. 4, 265-270.

25 Wanunu M \& Meller A (2007) Chemically modified solid-state nanopores. Nano Lett. 7, 1580-5.

26 Wei R, Gatterdam V, Wieneke R, Tampé R \& Rant U (2012) Stochastic sensing of proteins with receptor-modified solid-state nanopores. Nat. Nanotechnol. 7, 257-63.

27 Seeman NC (1982) Nucleic acid junctions and lattices. J. Theor. Biol. 99, 237-47. 
28 Rothemund PWK (2006) Folding DNA to create nanoscale shapes and patterns. Nature 440, 297302.

29 Kuzyk A, Schreiber R, Fan Z, Pardatscher G, Roller E-M, Högele A, Simmel FC, Govorov AO \& Liedl $T$ (2012) DNA-based self-assembly of chiral plasmonic nanostructures with tailored optical response. Nature 483, 311-4.

30 Thacker V V., Herrmann LO, Sigle DO, Zhang T, Liedl T, Baumberg JJ \& Keyser UF (2014) DNA origami based assembly of gold nanoparticle dimers for surface-enhanced Raman scattering. Nat. Commun. 5, 1-7.

31 Jahn K, Tørring T, Voigt NV, Sørensen RS, Bank Kodal AL, Andersen ES, Gothelf KV \& Kjems J (2011) Functional patterning of DNA origami by parallel enzymatic modification. Bioconjug. Chem. 22, 819-823.

32 Stein IH, Steinhauer C \& Tinnefeld P (2011) Single-molecule four-color FRET visualizes energytransfer paths on DNA origami. J. Am. Chem. Soc. 133, 4193-5.

33 Tørring T, Voigt N V, Nangreave J, Yan H \& Gothelf K V (2011) DNA origami: a quantum leap for self-assembly of complex structures. Chem. Soc. Rev. 40, 5636-5646.

34 Castro CE, Kilchherr F, Kim D-N, Shiao EL, Wauer T, Wortmann P, Bathe M \& Dietz H (2011) A primer to scaffolded DNA origami. Nat. Methods 8, 221-229.

35 Kuzuya A \& Komiyama M (2010) DNA origami: fold, stick, and beyond. Nanoscale 2, 310-22.

36 Simmel FC (2008) Three-dimensional nanoconstruction with DNA. Angew. Chemie Int. Ed. 47, 5884-7.

37 Douglas SM, Dietz H, Liedl T, Högberg B, Graf F \& Shih WM (2009) Self-assembly of DNA into nanoscale three-dimensional shapes. Nature 459, 414-8.

38 Dietz H, Douglas SM \& Shih WM (2009) Folding DNA into twisted and curved nanoscale shapes. Science 325, 725-730.

39 Han D, Pal S, Nangreave J, Deng Z, Liu Y \& Yan H (2011) DNA origami with complex curvatures in three-dimensional space. Science, 342-346.

40 Pinheiro A V, Han D, Shih WM \& Yan H (2011) Challenges and opportunities for structural DNA nanotechnology. Nat. Nanotechnol. 6, 763-72.

41 Bai X \& Martin T (2012) Cryo-EM structure of a 3D DNA-origami object. Proc. Natl. Acad. Sci. U. S. A. 109, 20012-20017.

42 Douglas SM, Marblestone AH, Teerapittayanon S, Vazquez A, Church GM \& Shih WM (2009) Rapid prototyping of 3D DNA-origami shapes with caDNAno. Nucleic Acids Res. 37, 5001-6.

43 Bell NAW, Engst CR, Ablay M, Divitini G, Ducati C, Liedl T \& Keyser UF (2012) DNA origami nanopores. Nano Lett. 12, 512-517. 
44 Wei R, Martin TG, Rant U \& Dietz H (2012) DNA origami gatekeepers for solid-state nanopores. Angew. Chemie Int. Ed. 51, 4864-7.

45 Hall AR, Scott A, Rotem D, Mehta KK, Bayley H \& Dekker C (2010) Hybrid pore formation by directed insertion of alpha-hemolysin into solid-state nanopores. Nat. Nanotechnol. 5, 874877.

46 Hernández-Ainsa S, Bell NAW, Thacker V V, Göpfrich K, Misiunas K, Fuentes-Perez ME, MorenoHerrero F \& Keyser UF (2013) DNA origami nanopores for controlling DNA translocation. ACS Nano 7, 6024-30.

47 Plesa C, Ananth AN, Linko V, Gülcher C, Katan AJ, Dietz H \& Dekker C (2014) lonic Permeability and Mechanical Properties of DNA Origami Nanoplates on Solid-State Nanopores. ACS Nano 8, 35-43.

48 Hernández-Ainsa S, Misiunas K, Thacker V V, Hemmig EA \& Keyser UF (2014) Voltage-Dependent Properties of DNA Origami Nanopores. Nano Lett. 14, 1270-1274.

49 Langecker M, Arnaut V, Martin TG, List J, Renner S, Mayer M, Dietz H \& Simmel FC (2012) Synthetic lipid membrane channels formed by designed DNA nanostructures. Science 338, 9326.

50 Burns JR, Stulz E \& Howorka S (2013) Self-assembled DNA nanopores that span lipid bilayers. Nano Lett. 13, 2351-6.

51 Burns JR, Göpfrich K, Wood JW, Thacker V V., Stulz E, Keyser UF \& Howorka S (2013) Lipid-BilayerSpanning DNA Nanopores with a Bifunctional Porphyrin Anchor. Angew. Chemie Int. Ed. 52, 12069-12072.

52 Rotem D, Jayasinghe L, Salichou M \& Bayley H (2012) Protein detection by nanopores equipped with aptamers. J. Am. Chem. Soc. 134, 2781-7.

53 Li W, Bell NAW, Hernández-Ainsa S, Thacker V V, Thackray AM, Bujdoso R \& Keyser UF (2013) Single protein molecule detection by glass nanopores. ACS Nano 7, 4129-34.

54 Plesa C, Kowalczyk SW, Zinsmeester R, Grosberg AY, Rabin Y \& Dekker C (2013) Fast translocation of proteins through solid state nanopores. Nano Lett. 13, 658-63.

55 Jonsson M \& Dekker C (2013) Plasmonic Nanopore for Electrical Profiling of Optical Intensity Landscapes. Nano Lett. 13, 1029-1033.

56 Ke Y, Voigt N V, Gothelf K V \& Shih WM (2012) Multilayer DNA origami packed on hexagonal and hybrid lattices. J. Am. Chem. Soc. 134, 1770-4.

57 Keyser U, Koeleman B, Dorp S Van, Krapf D, Smeets RMM, Lemay SG, Dekker NH \& Dekker C (2006) Direct force measurements on DNA in a solid-state nanopore. Nat. Phys. 2, 473-477.

58 Langecker M, Arnaut V, List J \& Simmel FC (2014) DNA Nanostructures Interacting with Lipid Bilayer Membranes. Acc. Chem. Res. doi: 10.1021/ar500051r 
59 Stoddart D, Ayub M, Höfler L, Raychaudhuri P, Klingelhoefer JW, Maglia G, Heron A \& Bayley H (2014) Functional truncated membrane pores. Proc. Natl. Acad. Sci. U. S. A. 111, 2425-30.

60 Thompson JR, Cronin B, Bayley H \& Wallace MI (2011) Rapid assembly of a multimeric membrane protein pore. Biophys. J. 101, 2679-83.

$61 \mathrm{Gu} H$, Chao J, Xiao S-J \& Seeman NC (2010) A proximity-based programmable DNA nanoscale assembly line. Nature 465, 202-5.

62 Andersen ES, Dong M, Nielsen MM, Jahn K, Subramani R, Mamdouh W, Golas MM, Sander B, Stark H, Oliveira CLP, Pedersen JS, Birkedal V, Besenbacher F, Gothelf K V \& Kjems J (2009) Selfassembly of a nanoscale DNA box with a controllable lid. Nature 459, 73-76.

63 Douglas SM, Bachelet I \& Church GM (2012) A Logic-Gated Nanorobot for Targeted Transport of Molecular Payloads. Science 335, 831-834.

64 Villar G, Graham AD \& Bayley H (2013) A tissue-like printed material. Science 340, 48-52. 